\title{
ON THE EXTERIOR CRACK WITH CONTACT ZONES
}

\author{
JOHN DUNDURS \\ Department of Civil Engineering, Northwestern University, Evanston, IL 60201, U.S.A. \\ and \\ MARIA COMNINOU \\ Department of Civil Engineering, University of Michigan, Ann Arbor, MI 48109, U.S.A. \\ (Communicated by I. N. SNEDDON)
}

Abstract-The paper discusses the exterior crack with possible contact zones, and shows by an example
that contact between the crack faces exists for most combinations of the stress resultants applied at infinity.

\section{INTRODUCTION}

Iт Is generally recognized, even if scant attention is paid to the requirement in practice, that all elasticity solutions of cracks must be checked for certain inequalities. This is due to the fact that in analyzing a crack under general conditions of loading, it is not known in advance whether the crack is fully open or whether its faces make contact. In the open part of the crack, the gap between the crack faces may not be negative and, if the crack faces are indeed in contact, the contact tractions may not be tensile. The solutions for cracks with negative gaps embedded in the literature may not be entirely useless as they can sometimes be used in superposition if the total load opens the crack completely. It must be noted, however, that there may not be any loads that open the crack completely and some contact between the crack faces always exists, as exemplified by interface cracks [1] and cracks that touch an interface [2]. Nonetheless the fundamental elasticity solutions for cracks should be reviewed and, if need be, recast in a unilateral framework ensuring that the inequalities are satisfied. This is especially desirable for the plane problem of the exterior crack in an unbounded solid, as there still appears to be some question about the proper boundary conditions at infinity [3], which in turn are connected with the possible contact between the crack faces.

It will be assumed here that the contact between the crack faces is frictionless.

\section{MATHEMATICAL DESCRIPTION OF THE PROBLEM}

We consider the problem of the exterior crack in a formulation that leads to singular integral equations. For this purpose the solid containing the two-sided exterior crack (see Fig. 1) is first cut into two half planes, and each half plane is subjected to the desired loads. The loads may be applied to the crack faces outside the interval $|x|<a$, where the two half planes were joined before cutting, or at interior points. The loads may have a finite resultant on each of the half planes, they may be self-equilibrated or, for that matter, the elastic fields may be induced by suitably specified inelastic strains. If either of the half planes is subjected to loads with a resultant, equilibrium is provided by tractions at large distances, as it can be deduced from the known Green's function for an elastic half plane [4]. The order of the stresses at infinity also is known from the Green's function $\left(O\left(r^{-1}\right)\right.$ for resultant forces, $O\left(r^{-2}\right)$ for resultant couples and inelastic strains with a net force doublet and $o\left(r^{-2}\right)$ for loads containing neither a force, couple or force doublet).

Next the continuity of the material in the neck section $|x|<a$ is restored by applying to both half planes on this interval normal and shearing tractions that satisfy Newton's third law. As this is done it is possible that contact zones develop and the crack faces exert compressive tractions on each other outside the interval $|x|<a$. The two half planes can be joined in such a way that the tractions in the neck section together with those in the contact intervals are self-equilibrated and the stress resultants at infinity remain unaffected. It is also possible, however, to adjust the tractions used in joining the half planes so that the stress resultants acting on each of the half planes at infinity are changed by the same amount. 


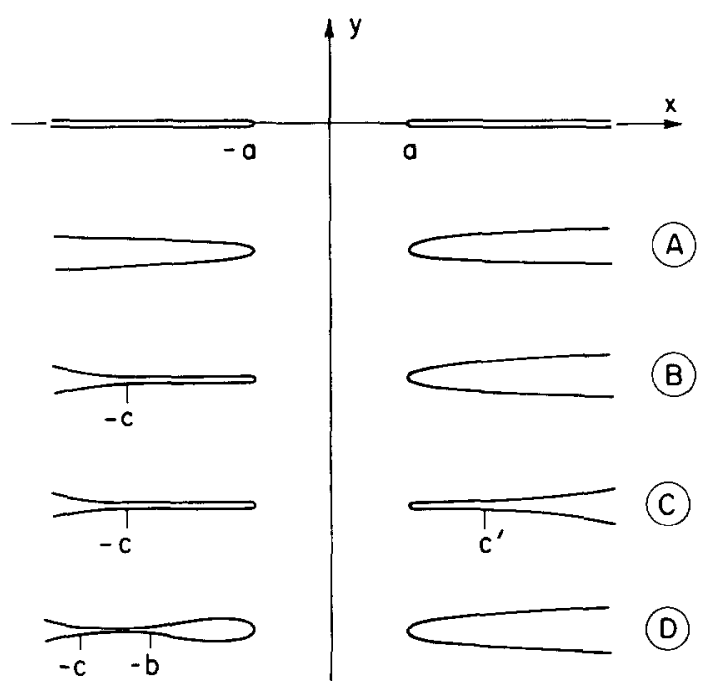

Fig. 1. The exterior crack under different conditions. A: open-open, B: closed-open, C: closed-closed, D: detached contact.

The two key quantities in the formulation of the problem are the gap

$$
g(x)=u_{y}(x, 0+)-u_{y}(x, 0-)
$$

and the tangential shift

$$
h(x)=u_{x}(x, 0+)-u_{x}(x, 0-)
$$

between the surfaces of the two half planes. If $g_{0}(x)$ and $h_{0}(x)$ denote the gap and the shift developed during loading when the half planes are disconnected, and $g_{1}(x)$ and $h_{1}(x)$ those caused by the surface tractions during the joining of the half planes, the conditions to be satisfied are

$$
\begin{gathered}
g(x)=g_{0}(x)+g_{1}(x)+\alpha+A x=0, \quad|x|<a \text { and } x \in C \\
h(x)=h_{0}(x)+h_{1}(x)+\beta=0, \quad|x|<a
\end{gathered}
$$

where $C$ is the union of the contact intervals outside $|x|<a$, and the free constants $\alpha, \beta$ and $A$ are associated with arbitrary rigid body displacements of the half planes. The conditions (3) and (4) can be differentiated without loss of generality provided appropriate considerations are introduced in case of disjoint contact zones, and

$$
\begin{gathered}
\frac{\mathrm{d} g_{0}(x)}{\mathrm{d} x}+\frac{\mathrm{d} g_{1}(x)}{\mathrm{d} x}+A=0, \quad|x|<a \text { and } x \in C \\
\frac{\mathrm{d} h_{0}(x)}{\mathrm{d} x}+\frac{\mathrm{d} h_{1}(x)}{\mathrm{d} x}=0, \quad|x|<a .
\end{gathered}
$$

It is readily derived from the Flamant solution[5] that

$$
\begin{aligned}
& \frac{\mathrm{d} g_{1}(x)}{\mathrm{d} x}=-\frac{\kappa+1}{2 \pi \mu} \int_{-\infty}^{\infty} \frac{F_{\mathrm{y}}(\xi) \mathrm{d} \xi}{\xi-x} \\
& \frac{\mathrm{d} h_{1}(x)}{\mathrm{d} x}=-\frac{\kappa+1}{2 \pi} \int_{-\infty}^{\infty} \frac{F_{x}(\xi) \mathrm{d} \xi}{\xi-x}
\end{aligned}
$$


where $F_{x}$ and $F_{y}$ denote the components of surface tractions developed in joining the half planes (the sign convention for $F_{x}$ and $F_{y}$ is the same as for the stress components $\sigma_{x y}$ and $\sigma_{y y}$ ). Thus (5) and (6) lead to the integral equations

$$
\begin{gathered}
\left\{\int_{-a}^{a}+\int_{C}\right\} \frac{F_{y}(\xi) \mathrm{d} \xi}{\xi-x}=\frac{2 \pi \mu}{\kappa+1}\left\{\frac{\mathrm{d} g_{0}(x)}{\mathrm{d} x}+A\right\}, \quad x<|a| \text { and } x \in C \\
\int_{-a}^{a} \frac{F_{x}(\xi) \mathrm{d} \xi}{\xi-x}=\frac{2 \pi \mu}{\kappa+1} \frac{\mathrm{d} h_{0}(x)}{\mathrm{d} x}, \quad|x|<a
\end{gathered}
$$

The inequalities the solution must satisfy are

$$
\begin{aligned}
& g(x)>0, \quad|x|>a \text { and } x \notin C \\
& F_{y}(x)<0, \quad x \in C .
\end{aligned}
$$

Of particular interest is the resultant of the tractions transmitted between the two half planes. If the resolution is done at the origin, the force and the couple are

$$
\begin{gathered}
R=\int_{-\infty}^{\infty} F_{y}(x) \mathrm{d} x \\
M=\int_{-\infty}^{\infty} x F_{y}(x) \mathrm{d} x .
\end{gathered}
$$

If the loads that cause the deformations of the half planes while they are detached are self-equilibrated on each of them, $R$ and $M$ are simply the force and couple applied at infinity. Otherwise, $R$ and $M$ are the imposed changes in these quantities.

As the unknown positions and extents of the contact zones depend on the deformed shapes of the half planes and the additional deformations induced by joining, little more can be said about the problem in general terms. Consequently we concentrate on an example for which the contact zones are adjacent to the neck or, if detached, are in its vicinity.

\section{EXAMPLE}

The simplest example is obtained by taking

$$
\begin{gathered}
g_{0}(x)=\frac{1}{2} k x^{2}, \quad(k>0) \\
h_{0}(x)=0
\end{gathered}
$$

which corresponds to the applied loads causing constant convex curvatures of the half-plane surfaces. It may be noted that constant curvatures would be induced by a bending-type loading of the half planes or inelastic strains that do not depend on the $x$ coordinate. Moreover the example can be viewed as derived from the Hertz contact problem: Two cylindrical bodies are pressed together to develop contact on the interval $|x|<a$, the contact is welded, and subsequently the cylinders are subjected to different loads. The example also provides a one-term approximation for a more general type of $g_{0}(x)$ when the third term in its Taylor expansion does not vanish (constant and linear terms in $g_{0}(x)$ are of no consequence as they correspond to rigid body displacements).

Elasticity problems with unilateral constraints involve not only inequalities but also unknown intervals on which the different boundary conditions hold, and their solution requires that the general arrangement of those intervals be anticipated at the outset. In the present example it is immediately expected that the following cases can arise (see Fig. 1):

1. The crack is open at both tips, and there are no contact zones (open-open). 
2. The left tip of the crack is closed so that there is an adjacent contact zone, but the right tip is open (closed-open).

3. Both tips of the crack are closed and adjacent contact zones exist (closed-closed).

The case of the open-closed crack need not be considered separately in this example because of symmetry. It will be discovered in the course of solution of these cases, however, that another possibility exists:

4. The crack is open at both tips, but there is a separate contact zone (detached contact).

The integral equation is the same for all cases, and

$$
\int_{L} \frac{F_{y}(\xi) \mathrm{d} \xi}{\xi-x}=\frac{2 \pi \mu}{\kappa+1}(k x+A), \quad x \in L
$$

where $L$ is the union of intervals on which the two half planes transmit normal tractions. However, the nature of the admissible solutions is different from one case to another. Since no net forces or couples act on the half planes while they are detached, the force and couple at infinity are in this example

$$
\begin{aligned}
R^{\infty} & =\int_{L} F_{y}(x) \mathrm{d} x \\
M^{\infty} & =\int_{L} x F_{y}(x) \mathrm{d} x .
\end{aligned}
$$

\section{Open-open case}

The integral equation for this case holds on the interval $|x|<a$, and the physically appropriate solution is singular at $x= \pm a$. Thus [6]

$$
F_{y}(x)=\frac{2 \mu}{\kappa+1} \frac{1}{\left(a^{2}-x^{2}\right)^{1 / 2}}\left\{k\left(x^{2}-\frac{1}{2} a^{2}\right)+A x+B\right\}, \quad|x|<a
$$

where $B$ is a free constant. The force and couple at infinity are from (18) and (19)

$$
R^{\infty}=\frac{2 \pi \mu}{\kappa+1} B, \quad M^{\infty}=\frac{\pi \mu a^{2}}{\kappa+1} A .
$$

Consequently, the two free constants are determined by $R^{\infty}$ and $M^{\infty}$, and

$$
F_{y}(x)=\frac{1}{\pi\left(a^{2}-x^{2}\right)^{1 / 2}}\left\{R^{\infty}+\frac{2 M^{\infty}}{a} \frac{x}{a}+\frac{\pi \mu k}{\kappa+1}\left(2 x^{2}-a^{2}\right)\right\}, \quad|x|<a .
$$

It is clear that these results cease to be valid when the stress intensity factors become negative, because singular compressive tractions are associated with overlapping of material near the crack tips [7]. The inequalities $K(x=+a)>0$ and $K(x=-a)>0$ yield from (22)

$$
\begin{gathered}
\frac{M^{\infty}(\kappa+1)}{\pi \mu k a^{3}}>-\frac{R^{\infty}(\kappa+1)}{2 \pi \mu k a^{2}}-\frac{1}{2} \\
\frac{M^{\infty}(\kappa+1)}{\pi \mu k a^{3}}<\frac{R^{\infty}(\kappa+1)}{2 \pi \mu k a^{2}}+\frac{1}{2}
\end{gathered}
$$

which restrict the points in the $R^{\infty}, M^{\infty}$ plane to the wedge-shaped region indicated in Fig. 2 (because of symmetry only positive values of $M^{\infty}$ need to be shown). Conditions (23) and (24) do not guarantee, however, that the gap between the crack faces is positive everywhere on $|x|>a$. The expression for the gap between the crack faces, derived from (3) and (7) using (22), is

$$
g(x)=\frac{1}{2} k a^{2} \operatorname{sgn} x\left\{\hat{R} \log \left|\frac{x}{a}+\left(\frac{x^{2}}{a^{2}}-1\right)^{1 / 2}\right|+2 \hat{M}\left(\frac{x^{2}}{a^{2}}-1\right)^{1 / 2}+\frac{x}{a}\left(\frac{x^{2}}{a^{2}}-1\right)^{1 / 2}\right\}, \quad|x|>a
$$




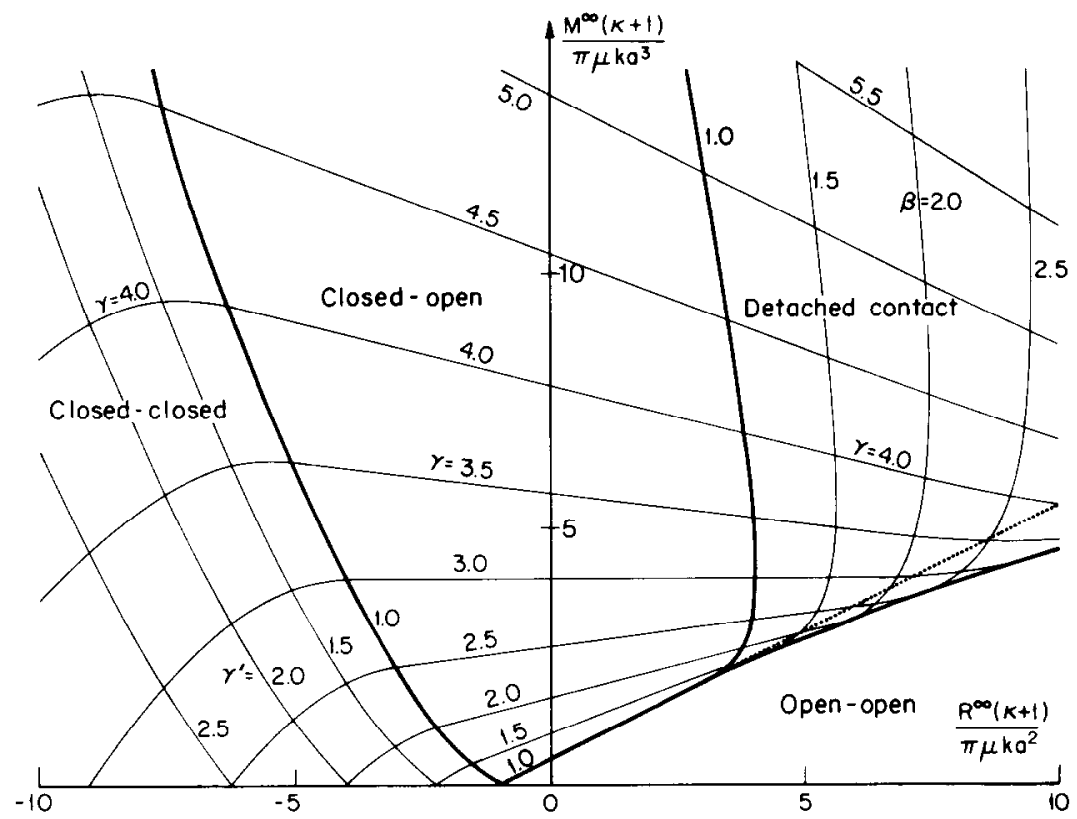

Fig. 2. The different regimes in the $R^{\infty}, M^{\infty}$ plane. $\beta=b / a, \gamma=c / a$ and $\gamma^{\prime}=c^{\prime} / a$.

where

$$
\hat{R}=\frac{R^{\infty}(\kappa+1)}{\pi \mu k a^{2}}, \quad \hat{M}=\frac{\hat{M}^{\infty}(\kappa+1)}{\pi \mu k a^{3}}
$$

Because of symmetry, we need to consider only the left side of the crack $(x<-a)$. It follows from (25) that for $\hat{M}>2$ the gap has zero slope at two locations defined by

$$
x_{1,2}=-\frac{1}{2} a\left\{\hat{M} \pm\left[\hat{M}^{2}-2(\hat{R}-1)\right]^{1 / 2}\right\}, \quad x_{2}>x_{1}
$$

as shown in Fig. 3. Overlapping of the crack faces occurs for $g\left(x_{1}\right)<0$.

Therefore, the equation

$$
g\left(x_{1}\right)=0
$$

defines the boundary in the $R^{\infty}, M^{\infty}$ plane inside which no overlap takes place at some finite distance from the crack tips. This boundary, which can be computed numerically and is shown in Fig. 2 , is in the range $\hat{M}>2$ more restrictive than that defined by (24).

\section{Closed-open case}

The integral eqn (17) is then valid on the interval $-c<x<+a$, and the appropriate solution must be bounded at $x=-c$ [7], but singular at $x=+a$. Hence [6]

$$
F_{y}(x)=\frac{2 \mu}{\kappa+1}\left(\frac{c+x}{a-x}\right)^{1 / 2}\left\{\frac{1}{2} k(2 x-a-c)+A\right\},-c<x<a .
$$

The force and couple transmitted by the neck and the contact zone are from (18) and (19)

$$
\begin{gathered}
R^{\infty}=-\frac{\pi \mu}{4(\kappa+1)}(a+c)\{k(3 c-a)-4 A\} \\
M^{\infty}=\frac{\pi \mu}{4(\kappa+1)}(a+c)\left\{k(c-a)^{2}+A(3 a-c)\right\} .
\end{gathered}
$$




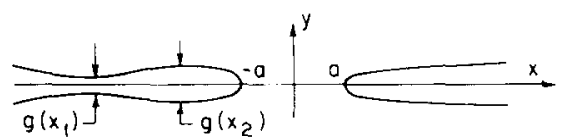

Fig. 3. Shape of the gap between the crack faces before development of a detached contact zone.

The boundaries in the $R^{\infty}, M^{\infty}$ plane of this regime are determined by the requirements that $c>a, K(x=+a)>0$ and that $F_{y}(x)<0$ on $-c<x<-a$. The condition $c=a$ yields from (30) and (31)

$$
\frac{M^{\infty}(\kappa+1)}{\pi \mu k a^{3}}=\frac{R^{\infty}(\kappa+1)}{2 \pi \mu k a^{2}}+\frac{1}{2}
$$

which is one of the boundaries for the open-open case. Setting $K(x=+a)=0$ in (29) leads to

$$
A=\frac{1}{2} k(c-a)
$$

which substituted into (30) and (31) gives

$$
\begin{gathered}
\frac{R^{\infty}(\kappa+1)}{\pi \mu k a^{2}}=-\frac{1}{4}(\gamma+1)^{2} \\
\frac{M^{\infty}(\kappa+1)}{\pi \mu k a^{3}}=\frac{1}{8}(\gamma+1)^{2}(\gamma-1)
\end{gathered}
$$

where

$$
\gamma=\frac{c}{a}
$$

With $\gamma$ serving as a parameter, (34) and (35) give a curve in the $R^{\infty}, M^{\infty}$ plane. Finally, it is seen from (29) that the requirement of compressive normal tractions in the contact interval $-c<x<-a$ is most critical at $x=-a$. Setting $F_{y}(-a)=0$, gives

$$
A=\frac{1}{2} k(3 a+c)
$$

which in turn substituted into (30) and (31) yields the boundary curve

$$
\begin{gathered}
\frac{R^{\infty}(\kappa+1)}{\pi \mu k a^{2}}=\frac{1}{4}(\gamma+1)(7-\gamma) \\
\frac{M^{\infty}(\kappa+1)}{\pi \mu k a^{3}}=\frac{1}{8}(\gamma+1)\left(11-4 \gamma+\gamma^{2}\right)
\end{gathered}
$$

with the parameter $\gamma$ defined by (36). The extent of the closed-open regime in the $R^{\infty}, M^{\infty}$ plane is shown in Fig. 2.

The loci of $\gamma=c / a=$ const in the $R^{\infty}, M^{\infty}$ plane are obtained by eliminating the constant $A$ between (30) and (31). Thus they are given by the expression

$$
\frac{M^{\infty}(\kappa+1)}{\pi \mu k a^{3}}=\frac{1}{4}(3-\gamma) \frac{R^{\infty}(\kappa+1)}{\pi \mu k a^{2}}+\frac{1}{16}(\gamma+1)^{3}
$$

and are also shown in Fig. 2. 


\section{Closed-closed case}

If there are contact zones at both tips of the crack, the integral eqn (17) holds on the interval $-c<x<c^{\prime}$ (see Fig. 1), and the solution must be bounded at both $x=-c$ and $x=+c^{\prime}$. This yields

$$
F_{y}(x)=-\frac{2 \mu k}{\kappa+1}(c+x)^{1 / 2}\left(c^{\prime}-x\right)^{1 / 2}, \quad-c<x<c^{\prime}
$$

The force and couple transmitted through the interval $-c<x<c^{\prime}$ are

$$
\begin{gathered}
R^{\infty}=-\frac{\pi \mu k\left(c+c^{\prime}\right)^{2}}{4(\kappa+1)} \\
M^{\infty}=-\frac{\pi \mu k\left(c+c^{\prime}\right)^{2}\left(c-c^{\prime}\right)}{8(\kappa+1)} .
\end{gathered}
$$

These results are identical to those one would get from the Hertz contact formulas upon a change of origin. The boundaries of this regime correspond to $c=a$ and $c^{\prime}=a$. For $c=a$

$$
\frac{R^{\infty}(\kappa+1)}{\pi \mu k a^{2}}=-\frac{1}{4}\left(\gamma^{\prime}+1\right)^{2} ; \quad \frac{M^{\infty}(\kappa+1)}{\pi \mu k a^{3}}=\frac{1}{8}\left(\gamma^{\prime}+1\right)^{2}\left(\gamma^{\prime}-1\right)
$$

with

$$
\gamma^{\prime}=\frac{c^{\prime}}{a}
$$

which is the same as one of the boundaries for the closed-open case.

The extent of this regime is shown in Fig. 2. Also shown in the figure are the loci of $\gamma=c / a=$ const and $\gamma^{\prime}=$ const which are obtained from (42) and (43). The condition $c^{\prime}=a$ gives a curve with negative values of $M^{\infty}$, and it need not be shown because of symmetry.

\section{Detached contact case}

It is seen from Fig. 2 that the previous three cases do not cover the whole $R^{\infty}, M^{\infty}$ plane, and that a fourth region remains. A clue as to what one should anticipate is provided by considering the tractions of the closed-open case for a choice of parameters that make the point in the $R^{\infty}, M^{\infty}$ plane fall in the fourth region. Taking for example $R^{\infty}(\kappa+1) / \pi \mu k a^{2}=5$ and $c=4 a(29)$ and (30) yield

$$
F_{y}(x)=\frac{2 \mu k a}{\kappa+1}\left(\frac{c+x}{a-x}\right)^{1 / 2}\left(\frac{x}{a}+\frac{5}{4}\right), \quad-4 a<x<a
$$

which shows that the contact zone $-4 a<x<-a$ would have to transmit tensile tractions on the interval $-5 a / 4<x<-a$. This suggests that for combinations of $R^{\infty}$ and $M^{\infty}$ falling in the fourth region one would get detached contact zones on the left side of the neck (see Fig. 1). Similarly, if we consider the open-open case, it is clear from Fig. 3 that a detached contact zone will develop near $x_{1}$ when $g\left(x_{1}\right)$ becomes negative.

Referring to the geometry of this case shown in Fig. 1, the solution of the integral eqn (17) is

$$
F_{y}(x)=-\frac{2 \mu}{\pi^{2}(\kappa+1)}\left|\frac{(x+b)(x+c)}{x^{2}-a^{2}}\right|^{1 / 2} \int_{L} \frac{k \xi+A}{\xi-x}\left|\frac{\xi^{2}-a^{2}}{(\xi+b)(\xi+c)}\right|^{1 / 2} \mathrm{~d} \xi, \quad x \in L
$$

where $L$ is the union of the intervals $(-c,-b)$ and $(-a, a)$. To ensure that the crack faces are actually in contact on the interval $(-c,-b)$ we must require that

$$
\int_{-a}^{-b} \frac{\mathrm{d} g(x)}{\mathrm{d} x} \mathrm{~d} x=0
$$




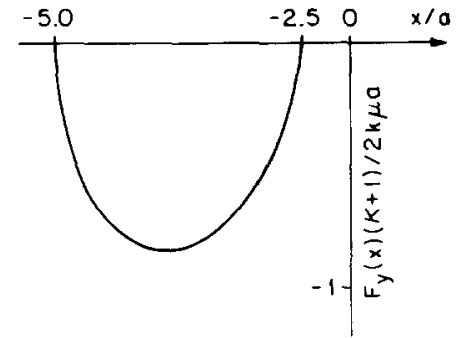

Fig. 4. Distribution of the contact tractions for $b / a=2.5$ and $c / a=5$.

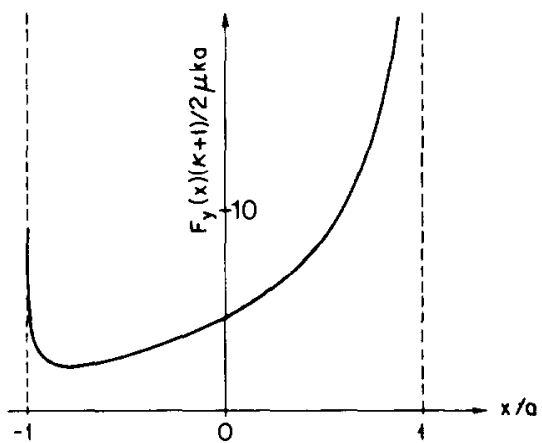

Fig. 5. Distribution of tensile tractions in the neck for $b / a=2.5$ and $c / a=5$.

Using (5), (7) and (15), this condition becomes in terms of $F_{y}(x)$

$$
\frac{\kappa+1}{2 \pi \mu} \int_{L} \log \left|\frac{\xi+a}{\xi+b}\right| F_{y}(\xi) \mathrm{d} \xi-\frac{1}{2} k\left(b^{2}-a^{2}\right)+A(b-a)=0 .
$$

The constant $A$ can be computed from (49) if $F_{y}(\xi)$ is eliminated by using (47). Thus, for chosen $b$ and $c, F_{y}(x)$ can be calculated by means of known quadratures [8] and the corresponding $R^{\infty}$ and $M^{\infty}$ then obtained from (18) and (19). This allows one to construct the loci of $\beta=b / a=$ const and $\gamma=c / a=$ const in the $R^{\infty}, M^{\infty}$ plane, which for selected values of the constants are shown in Fig. 2. It is interesting to note that the loci are tangent to the boundary curve between the open-open and detached contact regimes specified by (28). Moreover, the same curve on one side of the tangent point corresponds to $\gamma=$ const and on the other side to $\beta=$ const with the constant being the same. The boundary between the closed-open and detached contact regimes coincides with the locus of $\beta=1$.

The distribution of the contact tractions and the normal tractions in the neck are shown in Figs. 4 and 5 for $b / a=2.5$ and $c / a=5$ corresponding to $\hat{R}=9.44$ and $\hat{M}=8.86$.

\section{CONCLUSION}

The example discussed in detail shows quite clearly that the consideration of contact zones is particularly critical for the exterior crack, because it is not fully open for most combinations of the stress resultants applied at infinity.

Acknowledgement-Support by the Office of Naval Research through contract N00014-81-0626 is gratefully acknowledged.

\section{REFERENCES}

[1] M. COMNINOU and D. SCHMUESER, J. Appl. Mech. 46, 345 (1978).

[2] M. COMNINOU and J. DUNDURS, J. appl. Mech. 46, 97 (1979).

[3] M. P. STALLYBRASS, Int. J. Engng Sci. 19, 57 (1981)

[4] E. MELAN, Z. angew. Math. Mech. 12, 343 (1932).

[5] S. P. TIMOSHENKO and J. N. GOODIER, Theory of Elasticity, 3rd Edn McGraw-Hill, New York (1970).

[6] N. I. MUSKHELISHVILI, Singular Integral Equations. Noordhoff, Groningen (1953).

[7] J. DUNDURS and M. COMNINOU, J. Elasticity 9, 71 (1979).

[8] F. ERDOGAN, G. D. GUPTA and T. S. COOK, Methods of Analysis and Solution of Crack Problems (Edited by G. C. Sih), Noordhoff, Leyden (1973). 